\title{
Protocolo clínico como dispositivo analítico das relações de poder de profissionais de saúde
}

\author{
Clinical protocol as analytical dispositive of power relations among \\ health professionals
}

Tereza Cristina Peixoto', Maria José Menezes Brito ${ }^{2}$

\begin{abstract}
RESUMO Objetivou-se analisar as relações interprofissionais, tomando como referência o dispositivo protocolo clínico na terapia intensiva pediátrica. Estudo de caso qualitativo com coleta de dados feita por meio de observação, entrevistas com 14 profissionais e análise de discurso foucaultiana. Os protocolos clínicos regulam as relações, mas não asseguram a integração interprofissional. A clínica tradicional dificulta o lidar com a dimensão subjetiva implicada no sofrimento e morte, produzindo relações conflituosas, com busca pela perfeição e culpabilização por erros. Perante os conflitos, o protocolo multidisciplinar para cuidados paliativos pode favorecer a integração e ética da equipe.
\end{abstract}

PALAVRAS-CHAVE Relações interprofissionais; Unidades de Terapia Intensiva Pediátrica; Protocolos.

ABSTRACT This paper aimed at the analysis of inter-professional relationships, using as reference the clinical protocol applied in pediatric intensive care units. The study used qualitative methods based on observation, fourteen professional interviews and foucaultian discourse analysis. Data showed that the clinical protocols were able to regulate professional relationships but did not ensure inter-professional integration. The results indicate that traditional clinical practice hampers the ability to face the subjective dimension of suffering and death, thus producing conflicts, perfectionism and blaming. Before conflicts, the multidisciplinary palliative care protocols could help the building an integrated and ethic team.

KEYWORDS Interprofessional relations; Intensive Care Units, Pediatric; Protocols.

1 Centro Universitário (UNA) - Belo Horizonte (MG), Brasil.

terezacpc@hotmail.com

2 Universidade Federal de Minas Gerais (UFMG),

Departamento de

Enfermagem Aplicada -

Belo Horizonte, Brasil.

mj.brito@globo.com 


\section{Introdução}

Após a Segunda Guerra Mundial, as novas estratégias políticas criadas pelos Estados configuram a biopolítica, a qual consiste na articulação das tecnologias de governo com conhecimentos científicos com vistas a racionalizar e regular os fenômenos sociais. As novas políticas instituídas pelos Estados reorganizaram o modelo de atenção à saúde com foco na prevenção de doenças e no direito à saúde da população (FOUCAULT, 2008B). Assim, os mecanismos de manipulação social passaram a ser mais sutis, conquistando o consentimento da população e não mais a docilização das pessoas (PASSOS, 2012).

No campo da saúde, nos séculos XIX e $\mathrm{XX}$, os avanços científicos e tecnológicos acentuaram o desenvolvimento da medicina principalmente na cura e na prevenção de doenças. Nesse contexto, foram desenvolvidas intervenções tecnológicas sofisticadas, como, por exemplo, o Centro de Tratamento Intensivo, para pacientes em estado crítico. No Brasil, a partir dos anos 1970, tornam-se mais evidentes reivindicações sociais e políticas em prol da promoção da saúde, em defesa da vida e ampliação da cidadania, que se traduzem no movimento denominado como Reforma Sanitária.

$\mathrm{Na}$ atualidade, Ferreira Neto e Araújo (2014) mencionam a existência de distintos modelos de gestão e atenção e modos de relacionamento entre os sujeitos no trabalho em saúde. Trata-se do modelo que traduz a lógica da Reforma Sanitária e daquele que atende aos interesses econômicos neoliberais, com ênfase nas intervenções medicalizantes.

Cabe salientar que o modelo de gestão e atenção defendido pela Reforma Sanitária apresenta gestão democrática, com relações integradas no trabalho em equipe e em rede. Ademais, a Clínica Ampliada privilegia a participação do usuário no tratamento e considera a singularidade dos sujeitos, tendo como foco das ações a promoção da saúde e a Clínica Ampliada (BEDRIKOW; CAMPOS, 2011). Por outro lado, o modelo neoliberal, predominante nas práticas de saúde, dificulta os avanços do Sistema Único de Saúde (SUS), haja vista a centralização do saber médico, com ênfase na cura de doenças, a escassa participação do usuário, as ações fragmentadas e medicalizantes, caracterizando, pois, a clínica tradicional (FERREIRA NETO; ARAúJo, 2014). Para Bedrikow e Campos (2011), a clínica tradicional é eficaz para a cura de várias doenças, mas é frágil para lidar com demandas subjetivas.

Nesse cenário híbrido, as necessidades de aprimoramento na gestão e atenção na saúde pública se tornam mais complexas, em decorrência da exigência de qualidade com redução dos riscos assistenciais e controle financeiro. Além disso, para Bernardes et al. (2011), há a necessidade da criação de mecanismos para a transformação da cultura dos serviços em prol da integralidade assistencial e democratização da gestão. Nessa perspectiva, há que se chamar a atenção para o protocolo clínico como importante ferramenta de gestão voltada para o controle financeiro e redução dos riscos assistenciais. Sobre os protocolos voltados para a gestão da clínica, Mendes (2011) destaca a importância do envolvimento efetivo da equipe multidisciplinar na sua elaboração com a finalidade de integrar a equipe de saúde.

Apesar dos esforços voltados para a consolidação dos novos modelos de gestão e atenção e, ainda, do estímulo à elaboração de protocolos clínicos com a participação da equipe, observa-se a fragmentação das práticas assistenciais, com visíveis fragilidades no trabalho multidisciplinar. A esse respeito, Bernardes et al. (2011) afirmam que a implementação dos novos modelos ou ferramentas gerenciais não são suficientes para assegurar mudanças culturais nos serviços de saúde e, nem mesmo, a integração entre os profissionais, o que decorre da micropolítica das relações, caracterizada por conflitos e corporativismos profissionais.

A partir do contexto descrito, objetivou-se, por meio do presente estudo, analisar 
as relações interprofissionais, tomando como referência o dispositivo protocolo clínico em uma unidade de tratamento intensivo pediátrica.

Ressalta-se a existência de estudos no campo da saúde coletiva sobre a micropolítica e subjetividade no trabalho em função da importância de estratégias para a transformação da cultura no SUS e efetivação de seu modelo de atenção e gestão. Nessa direção, este estudo contribui para os demais, enfatizando a importância da atenção integral na Unidade de Terapia Intensiva Pediátrica (Utip), mesmo sendo destinado a procedimentos intervencionistas. A Clínica Ampliada na Utip contribui para a humanização da assistência e preparo dos profissionais no lidar com a dimensão subjetiva no envolvimento com as crianças e seus pais. Espera-se que este estudo contribua para a criação de estratégias para a produção de novas formas de relacionamento entre os sujeitos com foco na integralidade e na saúde dos profissionais.

\section{Percurso metodológico}

A concepção teórico-metodológica deste estudo é de natureza analítico-descritiva, com abordagem qualitativa (MINAYO, 2007). A estratégia de pesquisa foi o estudo de caso, por tratar-se de uma análise em profundidade de uma unidade em foco. Outro aspecto que caracteriza essa estratégia de pesquisa é a busca pela compreensão de 'como' e 'por que' ocorrem os fenômenos (DIONNE; LAVILLE, 1999).

O cenário de estudo foi uma Utip de um hospital universitário. A referida unidade foi escolhida por sua complexidade, uma vez que possui alta tecnologia e intensa demanda por acolhimento emocional de crianças e seus pais. A coleta de dados foi realizada por meio de observações participantes, no período de junho a agosto de 2014, em diversos horários de trabalho e em plantões de finais de semana, nos turnos da manhã, tarde e noite, perfazendo um total de 80 horas. As observações foram registradas em um diário de bordo e focalizaram as práticas e fluxos cotidianos do trabalho dos profissionais de saúde, as relações dos profissionais entre si, as relações desses com crianças e com familiares. Também foram utilizados como fonte de dados documentos e informações presentes no setor, sua organização arquitetônica e os acontecimentos diários. A pesquisadora estabeleceu uma relação próxima e afetiva com os sujeitos, profissionais, crianças e familiares.

A partir das observações, foram realizadas entrevistas com roteiro semiestruturado com um grupo heterogêneo de profissionais de saúde, escolhidos intencionalmente: 2 médicos, 1 enfermeira, 4 técnicas de enfermagem, 2 fisioterapeutas, 1 terapeuta ocupacional, 1 psicóloga e 1 nutricionista, 1 coordenador médico e 1 coordenador da enfermagem, totalizando 14 profissionais. As entrevistas foram concomitantes às observações.

As perguntas norteadoras das entrevistas foram: Como é para você trabalhar em uma Utip? Como é lidar com crianças doentes em uma Utip? Quais são os fatores que facilitam e dificultam o trabalho? Como são as relações interprofissionais? Como é o trabalho em equipe? Há reuniões de trabalho e como são? O que você pensa sobre os protocolos assistenciais? Por se tratar de um roteiro semiestruturado, outras perguntas foram elaboradas no decorrer das entrevistas, com vistas ao aprofundamento das questões sobre a temática em foco.

Os entrevistados foram esclarecidos sobre os objetivos da pesquisa e sobre seus riscos. O anonimato e o direito de interrupção das entrevistas foram assegurados e, no início das entrevistas, os participantes assinaram o Termo de Consentimento Livre e Esclarecido (TCLE), conforme resolução do Conselho Nacional de Saúde ${ }^{\circ}$ 466/12. O projeto foi aprovado pelo Comitê de Ética em Pesquisa da UFMG (Protocolo ETIC $n^{0}$ 30316914.0.0000.51.49) e pelo 
Comitê de Ética do hospital. Na apresentação dos resultados, os participantes foram identificados pela letra inicial de sua profissão a fim de se preservar seu anonimato.

Os dados das entrevistas foram submetidos à análise de discurso na perspectiva foucaultiana, identificando os enunciados, os quais consistem em acontecimentos que não estão dados, são produzidos por condições históricas, não são aparentes ou visíveis, mas também não estão ocultos nos atos de linguagem (PASSOS, 2012).

$\mathrm{Na}$ perspectiva analítica, assumem-se as práticas sociais e os saberes implicados nas relações de poder entre os indivíduos em um contexto sócio-histórico. Os resultados são apresentados com os seguintes subtítulos: Protocolo como regulador das relações de poder e cuidado de si e dos outros na Utip e cuidados paliativos.

\section{Resultados e discussão}

$\mathrm{Na}$ articulação entre relações de poder e saber, produzem-se as subjetividades, quer dizer, o modo como as pessoas se relacionam consigo mesmas e com os outros. Assumindo o dispositivo protocolo clínico, são analisados os regimes de verdade que sustentam as relações de poder entre profissionais de saúde, suas práticas discursivas e não discursivas. Considerou-se o dispositivo articulado aos três grandes eixos descritos por Foucault: o saber, o poder e a subjetividade.

O contexto sócio-histórico dos Centros de Tratamento Intensivo (CTIs) aponta que sua criação é produto do desenvolvimento da medicina intervencionista, com ênfase na patologia. O CTI surgiu na década de 1940, nos Estados Unidos da América e na Europa, no contexto de uma epidemia de poliomielite, para concentrar doentes críticos, com necessidades de intervenções terapêuticas invasivas, com monitoramento contínuo.

A denominação CTI refere-se ao agrupamento em uma mesma área física de mais de uma Unidade de Terapia Intensiva (UTI). A UTI, por sua vez, é uma área crítica destinada à internação de pacientes graves, com patologias ou faixa etária específicas. Esse setor é normatizado pela Agência de Vigilância Sanitária (Anvisa) (BRASIL, 2010).

Nos CTIs em geral, os saberes tecnocientíficos, a regulação dos procedimentos e o uso de protocolos clínicos são imprescindíveis para a eficiência e eficácia no trabalho. Ademais, o fato de a Utip, cenário da investigação, pertencer a um hospital universitário reforça a demarcação de um território com concentração de poder em torno do ensino e da ciência (CARMO ET AL., 2007). Na Utip em estudo, foram observadas disputas de saber entre pediatras intensivistas e outros especialistas.

Após a Constituição Federal de 1988 e a criação do SUS, os hospitais universitários sofreram mudanças na gestão para se integrarem à rede assistencial e atenderem aos compromissos pactuados com as instâncias gestoras do SUS, entre esses o uso de protocolos assistenciais (CARMO ET AL., 2007). Os protocolos clínicos ou assistenciais são recomendações para as práticas assistenciais baseados em evidência científica, elaborados de forma sistemática, a partir de orientações concisas sobre diagnóstico e tratamento. São importantes ferramentas gerenciais e para atualização clínica, sendo utilizados para reduzir a variação das ações na prática clínica por meio de normatizações (WERNECK; FARIA, 2009).

\section{Protocolo regulador das relações de poder}

Especificamente em relação ao cenário da pesquisa, observa-se que se trata de um setor com forte regulamentação por leis, normas e protocolos. Na entrevista, o médico 3, ao ser indagado sobre a observância dos protocolos, relata:

Quando eu falo dos protocolos, nossos protocolos são para nós mesmos seguirmos, né? Só 
os médicos. Então tem muita coisa assim para as outras clínicas. Tem poucos protocolos multidisciplinares. (M.3).

Essa enunciação explicita que os protocolos da Utip são fundamentados em conhecimentos médicos, ou seja, pertencem aos médicos. Esse fato confere lugar de poder e verdade no setor. Além disso, a escassez de protocolos multidisciplinares expressa a falta de participação efetiva da equipe na elaboração dos protocolos e o predomínio do saber médico.

Apesar de os protocolos serem médicos, os profissionais atribuem valor a seu uso, como no relato a seguir:

Eu sou suspeita, porque eu sou defensora máxima da padronização no Utip, não da mecanização da padronização. Quando você atende, você tem um protocolo de atendimento, esse é o protocolo, mas foge da mecanização, você faz um acalento na criança, você vê como ela está. (Fisio.2).

Nesse discurso, há uma defesa pela padronização como forma de organização do trabalho, mas sem mecanização dos procedimentos, trazendo à tona a polêmica sobre o uso dos protocolos e a objetivação de relações, contrários às discussões de humanização. Alguns estudos em UTI, como os de Araújo (2005), evidenciam que o uso de protocolos, normas e o lidar com máquinas complexas pelos trabalhadores influenciam as relações, que se tornam objetivadas entre os profissionais e os pacientes.

Alguns profissionais resistem ao uso de protocolos, por acreditarem que são dispensáveis para o exercício em sua área de atuação, como no relato a seguir:

[...] na verdade, a Terapia Ocupacional especificamente, por não ter obrigatoriedade na equipe. A gente está construindo os protocolos aos poucos. [...] assim, sinceramente, eu acho que as normas são importantes, mas é $[. .$.$] pra gente ter como$ consulta mesmo [...], mas eu acho que na questão da Terapia Ocupacional elas não sejam essenciais, imprescindíveis. (TO).

Esse relato defende a não obrigatoriedade da exclusividade dessa categoria profissional no setor, mas os profissionais da psicologia, nutrição e fonoaudiologia também não precisam ser exclusivos, são desejáveis na atenção aos pacientes, segundo as normatizações estabelecidas pela Agência Nacional de Vigilância Sanitária do SUS (Anvisa) para a autorização do funcionamento dos CTIs. Essa regulamentação fragiliza a integração desses profissionais na equipe e a incorporação de outros campos de conhecimentos relacionados à atenção integral nas discussões da equipe.

Ressalta-se que o fato desses profissionais não serem exclusivos no setor fragiliza a sua integração com os demais membros da equipe. Além disso, esses profissionais não se sentem reconhecidos, conforme o relato:

\section{A gente fica assim [...] não é reservado não, mas eles não reparam muito a nossa presen- ça. Então, na passagem de plantão, a gente não tem que estar sempre, de vez em quando a gente consegue acompanhar. (TO).}

Durante a observação, verificou-se que os profissionais da terapia ocupacional, da psicologia, da nutrição e da fonoaudiologia não participavam das reuniões na corrida de leitos. Esses profissionais não tinham seus protocolos concluídos e não os utilizavam na Utip, o que, na cultura da Utip, fragiliza ainda mais o reconhecimento de sua atuação, por ser um setor que valoriza práticas fundamentadas em evidência científica. A psicóloga relatou a esse respeito:

Olha, é [...] com relação a protocolo, a gente está mesmo formalizando, mas na Utip a gente não segue muito protocolo, é o trabalho mesmo de acompanhamento. (P.1). 
Nas observações, percebeu-se que as relações profissionais são reguladas pelas práticas cotidianas. Nesse sentido, a terapia ocupacional se relaciona prioritariamente com a psicologia, e a fisioterapia com a fonoaudiologia, enfermeiros, técnicos de enfermagem e médicos em decorrência do trabalho conjunto. A respeito da dificuldade de integração com os profissionais não exclusivos no setor, a Fisio (2) relatou:

É muito difícil, muito difícil, é por questões do número de profissionais, até da integração que esses outros profissionais têm aqui dentro, mas com a fonoaudiologia é mais tranquilo, a gente trabalha junto.

A integração profissional, em função da afinidade na prática de trabalho, não é suficiente para uma Clínica Ampliada, pois esta requer também a integração de campos de conhecimentos distintos, produzindo uma visão ampliada de saúde. A Clínica Ampliada, como afirmam Bedrikow e Campos (2011), considera a ampliação do objeto de intervenção, incluindo não só a doença, mas também o sujeito e seu contexto, sua forma de viver. Para tal, é necessária a participação dos sujeitos no tratamento e o trabalho em equipe multidisciplinar.

Em contraposição à perspectiva da Clínica Ampliada, a falta de reuniões multidisciplinares com a participação efetiva dos profissionais dificulta a integração e a visão ampliada sobre a saúde. Evidenciou-se que as reuniões multiprofissionais ocorrem diariamente, na passagem de plantão no turno da manhã, mas têm pouca participação da equipe, uma vez que possuem a finalidade de discutir casos mediados pelo discurso médico, direcionados para orientações clínicas. A respeito da participação nas reuniões, a psicóloga relatou:

Olha, atualmente, a gente não tem conseguido participar, aquela reunião é extremamente técnica, ah, o nivel de potássio é tanto, é uma reunião super-rápida, de passagem de plantão, em que os profissionais estão querendo ir embora, outros estão chegando. Então, comecei a participar um tempo, e não estava funcionando. (P.1).

Durante as observações, constatou-se que a fisioterapia participava mais das reuniões do que os demais profissionais. Essa proximidade é evidenciada na elaboração dos protocolos, uma vez que a fisioterapia elaborou alguns protocolos em parceria com os médicos, o que contribuiu para a integração de suas práticas, como no relato abaixo:

\footnotetext{
Aqui a gente tem alguns protocolos, já prontos, a fisioterapia e a equipe médica também têm muitos. No ano passado, eu até ajudei a montar dois desses protocolos. E a gente tenta colocar em prática, mas o trabalho todo ainda não está sistematizado. Eu acho que tinham que, de alguma forma, conscientizar as pessoas da importância disso, sabe? (F.2).
}

Esse discurso destaca as resistências dos profissionais com relação ao uso dos protocolos, que, nesse setor, são exigências dos médicos, deixando transparecer o conflito entre os profissionais.

No entanto, mesmo os protocolos da Utip sendo fundamentados no discurso médico, observa-se a resistência de alguns profissionais médicos em sua utilização, como no seguinte relato: "Os protocolos são seguidos, mas a gente ainda tem a cultura de achar que, o eu acho, tem muito do eu acho [...], mas isso está mudando" (M.1). O eu acho refere-se ao conhecimento não fundamentado em parâmetros científicos.

A resistência no seguimento de protocolos refere-se à resistência com a regulação das práticas clínicas (MENDES, 2011). No entanto, o seguimento dos protocolos confere valor perante conflitos interprofissionais e disputas de poder, como na relação entre médicos da Utip e os cirurgiões, como explicitado: Aí começou a aparecer outro ponto de conflito, que
antes era menos que [...] quando não tinha um
segmento da ação entre os médicos, o especialista
entrava aqui e determinava e fazia o que queria. A 
partir do momento que a equipe médica se organizou, é: você quer que faça isso com esse paciente, mas por quê? Cadê o protocolo disso? (M.3).

Percebe-se, por meio do discurso de M.3, que os protocolos legitimam as práticas e regulam as relações de poder. $\mathrm{Na}$ Utip, os médicos entrevistados relataram conflitos na definição de procedimentos com os especialistas, principalmente com os da cirurgia. Mediante esses conflitos, os protocolos fortalecem os pediatras em suas decisões terapêuticas.

Em outras situações, foram identificadas divergências entre profissionais quanto a procedimentos, e os protocolos asseguraram a decisão de quem os conhecia, como no relato dessa técnica de enfermagem:

Eu acho essenciais as normatizações, porque, quando existe um protocolo [...] quando há um protocolo, né? E, se eu não estou seguindo o protocolo que está escrito, o erro é meu. Quando o médico me cobra fora daquilo, ele não tem como cobrar, então, na realidade, o protocolo me respalda, excelente! Eu acho que tudo tem que ter um protocolo. (Tec.2).

Esse enunciado demonstra a importância dos protocolos para o posicionamento dos profissionais no setor e perante os conflitos.

Em diferentes ocasiões, foram observados médicos questionando a organização do trabalho de outros profissionais, principalmente das técnicas de enfermagem, explicitando os modos de relacionamento entre os sujeitos, como no seguinte relato:

Então, a gente ficou com um grupo novo de médicos, homogêneo, mas ainda um pouco mais estressado, exigente e, principalmente, exigente. Amenizou embates e brigas por coisas bobas, mas começou a ter um tipo de cobrança $[. .$.$] , mas$ se eu tenho meus protocolos e tenho uma assistência organizada, na hora que eu vou lá, e a parte do outro falha, não se consegue manter o protoco-

lo, isso gera briga na beira do leito. (M.3).

Esse discurso apresenta o valor atribuído aos protocolos para segurança das práticas e justifica os conflitos interprofissionais. Foi possível constatar, inclusive, a regulação do trabalho de enfermeiros por parte de médicos, como nessa afirmação de E.2:

Muitas vezes as pessoas não respeitam o que a gente fala, briga com você por causa de [...], mas eu estudei também, né? Eu estudei para ser enfermeira, né? Isso é muito ruim aqui dentro, não é levado em consideração que teve a implantação do check list na corrida de leito, que estressou todo mundo. O check list feito pelos médicos e é da enfermagem.

O predomínio da clínica tradicional no setor relacionado à necessidade dos saberes e decisões médicas fragiliza o reconhecimento dos demais profissionais. Carapinheiro (1998), em sua pesquisa em um hospital, evidenciou que a regulação do trabalho dos demais profissionais de saúde por parte dos médicos é comum no cotidiano dos hospitais e caracteriza as relações de poder. Os médicos, no hospital, possuem um autogoverno sobre suas ações, ao mesmo tempo em que interferem na atuação dos demais profissionais.

Perante esses conflitos, observou-se que a equipe de técnicos de enfermagem registra continuamente seus procedimentos, para se protegerem. O registro é outro mecanismo de regulação das relações, assim como os protocolos clínicos. A esse respeito, uma profissional relatou: "Nós registramos pouco até, deveríamos registrar mais, pois isso nos dá respaldo" (Tec.2). O respaldo refere-se novamente à defesa desses profissionais diante de possíveis acusações ou processos legais. 


\section{O cuidado de si e dos outros na Utip e cuidados paliativos}

Entende-se que o saber médico, predominante na organização, subsidia e regula as práticas de trabalho, produzindo modos de relacionamento entre os profissionais. Nessa perspectiva, os profissionais na Utip apresentam o discurso do autocuidado, na perspectiva da biopolítica: "Eu faço exercício físico, não fumo, não bebo, mas gosto muito de sair" (M.1). Identifica-se que os profissionais se referem aos cuidados com seus corpos orgânicos. A respeito do cuidado de si na atualidade, os estudos de Foucault (2004) sobre a produção de subjetividade desde a antiguidade apontam mudanças com relação ao modo como as pessoas entendem o cuidado de si ao longo da história. $\mathrm{Na}$ antiguidade, esse cuidado relacionava-se ao exercício reflexivo sobre si mesmo; no cristianismo, é associado a atitudes individualistas, valorizando os sacrifícios pessoais para a conquista da vida eterna. Com o apogeu da ciência, a ênfase do cuidado de si se torna a busca constante do conhecimento racional sobre si mesmo.

Ademais, os profissionais valorizam tanto os controles que seguem que parecem identificar-se com eles em seus relacionamentos, como neste relato: " $\mathrm{Eu}$ tenho essa coisa de perfeccionismo, temos isso. Um rigor excessivo, o rigor não é ruim, mas, em contrapartida, ele traz um pouco de neurose" (E.3). O perfeccionismo necessário na atividade de trabalho é incorporado às condutas do sujeito, mas representa um sofrimento mental, nesse discurso. Segundo o médico (2):

Às vezes, quinze minutos numa tomada de decisão, que você atrasa, faz a diferença; às vezes, numa parada cardíaca, segundos fazem a diferença. Então, eu acho que o próprio ambiente te cobra isso.
Outra profissional analisa o perfil dos profissionais do setor:

Aqui é muito tenso, né? Todos são muito perfeccionistas, porque eu também sou, eu tenho o meu, né? [...] eu tenho muita intolerância quando as meninas trabalham de qualquer jeito. (E.2).

O ritmo intenso de trabalho, as exigências de controle e a fragilidade do trabalho em equipe multiprofissional intensificam as dificuldades no lidar com falhas, com erros, e principalmente com a morte de crianças. Ante essas dificuldades, as relações tornam-se tensas, com culpabilização pelos erros e se tornam propícias aos conflitos, como relatado:

A equipe é muito exigente, muito envolvida, mas assim se envolve muito emocionalmente, às vezes, com os casos, a ponto de ser assim, quase igual a um parente seu, aquele paciente que está ali. Então isso é $[\ldots]$, mas, assim, é doente a ponto de ter que se afastar para dar um tempo, mas eu sei que aqui o sofrimento é muito grande e que alguns trocam plantão para não ficar naquele período. Outros sofrem muito e reagem de uma forma sendo mais exigente, cada um tem uma reação. (M.1).

Esse relato refere-se ao intenso envolvimento afetivo dos médicos com as crianças, como se fossem um parente, mas sem referências para lidar com a dimensão subjetiva implicada nos afetos mobilizados, o que produz sofrimento no trabalho ou comportamentos exigentes. Não só os médicos, mas também os demais profissionais se envolvem com as crianças e sofrem com algumas mortes que mobilizam a equipe. Outros estudos também evidenciam esse envolvimento dos profissionais na Utip com as crianças e com seus pais (PITTA, 1999).

O sofrimento despertado é projetado nas relações familiares e cotidianas dos profissionais, como mostra o próximo relato:

Eu sou uma pessoa que tem muito medo de morrer o tempo todo, e eu sou uma pessoa que, se 
minha filha tem um resfriado, eu choro. Porque a gente vê que uma infecção de garganta, às vezes, pode virar uma miocardite, você está me entendendo? A gente fica meio neurótico. (Tec.2).

O médico 1, em resposta ao modo como os médicos se preparam para lidar com o sofrimento provocado no trabalho, responde:

Ah! Não tem preparo não, a gente não. A gente faz reuniões na equipe médica, muito mais para discutir tratamento, mas para tratar da saúde mental não tem um tipo de abordagem, não tem discussão, não tem reunião com a psicologia. (M.1).

Esse relato reflete a negação da subjetividade no discurso médico que, mesmo perante a constatação do sofrimento dos profissionais, não dispõe de estratégias para a expressão de sentimentos e reflexão sobre a morte. Pitta (1999), em seus estudos sobre o lidar com dor e morte como ofício, também evidencia essas dificuldades dos profissionais em lidar com a morte de crianças.

A dificuldade em lidar com a morte e a fragilidade do trabalho em equipe multiprofissional são evidenciadas no seguinte discurso, em justificativa à falta de protocolo para os cuidados paliativos no setor:

Eu acho que não vai ter, não tem como você escrever um protocolo de cuidados paliativos aqui, tem como você ter uma linha de raciocínio. $\mathrm{Na}$ pediatria não tem essa discussão, você acha que o menino pode, e para a família também e para a mãe tem que investir, como em casos neurológicos, por exemplo. (M.3).

Essa enunciação traz à tona a discussão de temas da bioética sobre investimento na recuperação da saúde diante de quadros clínicos com acentuados comprometimentos, como, por exemplo, nos casos neurológicos, nos quais a criança pode ficar dependente eternamente de respiradores artificiais. Rego et al. (2008) afirmam que a bioética tem sido amplamente discutida a partir do crescimento de pesquisas e de práticas científicas que intervêm na vida dos sujeitos, muitas vezes desconsiderando seus valores, direitos e escolhas.

Outro profissional relatou sua indignação com a situação do não reconhecimento dos limites terapêuticos.

[...] tem hora que tem de saber a hora de parar, né? Até quando investir em uma situação que não é nada de eutanásia, não é nada de distanásia, não é nada disso, é tentar entender que tem um limite, né? (F.2).

O planejamento dos cuidados paliativos é fundamental em todos os níveis de atenção à saúde. Deve ser iniciado desde o diagnóstico de uma doença crônica fora de possibilidades terapêuticas até a fase terminal. A discussão em torno da bioética contribui para a formação ética dos profissionais de saúde, uma vez que participa da constituição da reflexividade e construção identitária dos profissionais (RAMOS; DO Ó, 2009).

Para ampliar a discussão da bioética, é importante uma política que articule esses cuidados em todos os níveis de atenção, incluindo as Utips. Os cuidados paliativos são ações que proporcionam conforto e apoio aos pacientes e familiares no processo de doença até a fase final da vida, contemplando os aspectos psicossociais e espirituais, relacionados aos princípios do SUS. Não são ações para apressar ou adiar a morte. Salienta-se que os profissionais de saúde necessitam ser capacitados para esses cuidados (MENDES; VASCONCELLOS, 2015). As discussões a respeito dos cuidados paliativos podem estimular expressões de sentimentos e novas reflexões para a integralidade assistencial e para a saúde dos profissionais.

Entende-se, de acordo com Foucault (2008A), que o poder da ciência, estimulado pelo discurso biopolítico de intervir sobre a vida, prolongando-a, distancia cada vez mais os profissionais da saúde e 
a população de conceberem a morte como parte inerente à vida. Assim, a morte passa a ser mais proibida de ser dita do que a sexualidade na atualidade. Em função dessa negação, muitas vezes, intervenções médicas prolongam vidas sem analisarem as possibilidades de vida humana digna.

Pereira (2006) descreve que o surgimento da especialidade da pediatria foi impulsionado pela biopolítica, atendendo às intervenções em prol da redução da taxa de mortalidade infantil, que representam qualidade de vida da população em geral. Em pesquisa sobre a história da pediatria no Brasil, Pereira (2006, P. 2) defende que

No limite, a pediatria na história não fala da morte infantil, porque excluiu de seu campo de formação e de prática o enfrentamento reflexivo e a simbologia dos limites de seu saber.

A dificuldade em lidar com limites nas práticas e com a morte exacerba o sofrimento no trabalho dos profissionais e os conflitos em função de culpabilização pelos erros. O médico 1 , em resposta a como se sente após uma morte inesperada na Utip, argumenta:

[...] eu fico assim, com aquele sentimento de culpa, será que eu fiz tudo pelo meu paciente. Não, agora, assim eu estudo, fiz tudo que era para ter feito, fico assim com esse sentimento.

Esse relato traz à tona o sentimento de responsabilidade pela morte da criança, como se o profissional tivesse o controle sobre a vida. O lidar em um setor com recursos sofisticados para intervenção e prolongamento da vida parece produzir um imaginário controle sobre a vida por parte dos profissionais, principalmente dos médicos. As dificuldades vivenciadas nesse contexto são projetadas nas relações entre os profissionais, principalmente na relação entre os médicos e a enfermagem.

Salienta-se que o grupo de profissionais da enfermagem se encontra mais vulnerável, tanto pelo processo de trabalho, que envolve um lidar contínuo com crianças instáveis, como também por se tratar de um grupo numeroso e heterogêneo de profissionais com diferentes níveis de formação. Além disso, na ocasião da pesquisa, o hospital tinha realizado um concurso público para regularizar os contratos precários de trabalho, pois havia diferentes vinculações.

\section{Conclusão}

A Utip em estudo é uma unidade que se caracteriza pelo saber especializado, médico centrado, altamente tecnologizado, regulado por normas, leis e protocolos. A organização do serviço reproduz a fragmentação da assistência, pela dificuldade do convívio com os profissionais da psicologia e da terapia ocupacional. Evidenciou-se que, a despeito de os protocolos clínicos regularem as relações entre os profissionais e se traduzirem em qualidade assistencial para os profissionais, estes não asseguram a integração da equipe.

$\mathrm{Na}$ micropolítica das relações, identifica-se a produção de subjetividades por meio dos saberes biomédicos, caracterizadas como perfeccionistas, com dificuldades em lidar com a falta de controle, com o afeto despertado pelas crianças e seus pais e com a morte. Parte-se do entendimento de que a clínica tradicional não oferece saberes e práticas para lidar com a dimensão subjetiva, reduzindo-a a noção de sujeito estritamente racional. A noção de sujeito da Clínica Ampliada é de sujeito habitado pelo não saber, constituído sócio-historicamente, e em permanente construção, sendo importante a reflexividade sobre si mesmo para o cuidado de si e a busca de transformação. Esse é um trabalho ético defendido por Foucault (2004).

Salienta-se, por meio dos estudos sobre a bioética, a potência dos protocolos multidisciplinares para os cuidados paliativos 
para integração da equipe e formação ética dos profissionais, uma vez que abre espaço para a expressão de sentimentos e reflexões sobre a morte e sobre o papel de cada profissional na assistência. Ressalta-se que o presente estudo se restringiu a uma unidade de internação pediátrica, sendo necessárias novas pesquisas sobre a micropolítica em outros hospitais e setores.

\section{Referências}

ARAÚJO, L. F. S. Processos de subjetivação inscritos na constituição da experiência de si da/o enfermeira/o nas práticas assistenciais de um cenário de trabalho exemplar: a Unidade de Terapia Intensiva. 2005. 234 f. Tese (Doutorado em Enfermagem) Escola de Enfermagem, Universidade de São Paulo, Ribeirão Preto, 2005.

BEDRIKOW, R.; CAMPOS, G. W. S. Clínica: a arte de equilibrar a doença e o sujeito. Revista da Associação Médica Brasileira, São Paulo, v. 57, p. 610-613, 2011.

BERNARDES, A. et al. Modelo de gestão colegiada e descentralizada em hospital público: a ótica da equipe de enfermagem. Rev. Latino-Am. Enfermagem, Ribeirão Preto, v. 19, n. 4, p. 1003-10, 2011. Disponível em: <http://www.scielo.br/pdf/rlae/v19n4/pt_20.pdf >. Acesso em: 2 out. 2013

BRASIL. Resolução $n^{\circ}$ 7, de 24 de fevereiro de 2010. Dispõe sobre os requisitos mínimos para funcionamento de Unidades de Terapia Intensiva e dá outras providências. Brasília, DF: Ministério da Saúde, 2010. Disponível em: <http://http://www. anvisa.gov.br/hotsite/segurancadopaciente/documentos/rdcs/RDC\%20N\%C2\%BA\%207-2010.pdf>. Acesso em: 20 ago. 2014.

CARAPINHEIRO, G. Saberes e poderes no hospital: uma sociologia dos serviços hospitalares. Porto: Edições Afrontamento, 1998.

CARMO, M.; ANDRADE, I. G.; MOTA, J. A. C. Hospital universitário e gestão do sistema de saúde: uma trajetória positiva de integração. Revista Mineira de Enfermagem, Belo Horizonte, v. 11, n. 4, p. 387-394, jan./mar. 2007.

\section{Agradecimentos}

À Coordenação de Aperfeiçoamento de Pessoal de Nível Superior (Capes), fundação do Ministério da Educação (MEC) e ao Conselho Nacional de Desenvolvimento Científico e Tecnológico (CNPq) pelo investimento na formação em doutorado sanduíche na Universidade de Lisboa.
DIONNE, J.; LAVILLE, C. A Construção do saber: manual de metodologia de pesquisa em ciências humanas. Belo Horizonte: UFMG, 1999.

FERREIRA NETO, J. L.; ARAÚJO, G. J. N. Gestão e subjetividade no SUS: o enfrentamento de impasses em tempos neoliberais. Psicologia \& Sociedade, Belo Horizonte, v. 26, n. 3, p. 675-684, 2014.

FOUCAULT, M. Hermenêutica do sujeito. São Paulo: Martins Fontes, 2004.

Nascimento da Biopolítica. São Paulo: Martins

Fontes, 2008a.

Segurança, território, população. São Paulo:

Martins Fontes, 2008b.

MENDES, E. C.; VASCONCELLOS, L. C. F. Cuidados paliativos no câncer e os princípios doutrinários do SUS. Saúde em Debate, Rio de Janeiro, v. 39, n. 106, p. 881-892, set. 2015.

MENDES, V. E. As Redes de Atenção à Saúde. Brasília, DF: Organização Pan-Americana de Saúde, 2011.

MINAYO, M. C. S. (Org.). Pesquisa social: teoria, método e criatividade. Petrópolis: Vozes, 2007.

PASSOS, I. C. F. A noção de discurso em Michel Foucault. In: UNIVERSIDADE FEDERAL DE MINAS GERAIS. PET-Ciências Sociais (Org.). O poder em perspectiva. Belo Horizonte: Sografe, 2012. p. 79-88.

PEREIRA, J. S. História da Pediatria no Brasil de final do século XIX a meados do século XX. 2006. $2011 \mathrm{f}$. Tese 
(Doutorado em História) - Universidade Federal de Minas Gerais, Belo Horizonte, 2006.

PITTA, A. Hospital: dor e morte como ofício. São Paulo: Hucitec, 1999.

RAMOS, F. R. S.; DO Ó, J. Bioética e identidade profissional: a construção de uma experiência de si do trabalhador da saúde. Interface, Botucatu, v.13, n. 29, p. 259-70, abr./jun. 2009.

REGO, S.; GOMES, A. P.; SIQUEIRA-BATISTA, R. Bioética e humanização como temas transversais na formação médica. Rev. bras. educ. med., Rio de Janeiro, v. 32, n. 4, dez. 2008. Disponível em: <http:// www.scielo.br/pdf/rbem/v32n4/v32n4all.pdf>.

Acesso em: 8 abr. 2015.

WERNECK, M. A. F.; FARIA, H. P.; CAMPOS, K. F. C. Protocolo de cuidados à saúde e de organização do serviço. Belo Horizonte: Coopmed, 2009.

Recebido para publicação em abril de 2015

Versão final em setembro de 2015

Conflito de interesses: inexistente

Suporte financeiro: não houve 